\title{
MODEL ATMOSPHERES AND SPECTRAL ANALYSES OF
}

\section{WOLF-RAYET STARS}

\author{
W.-R. HAMANN \\ Institut für Theoretische Physik und Sternwarte der Universität Kiel, D-24098 Kiel, \\ B.R. Deutschland
}

\begin{abstract}
Stratified non-LTE models for expanding atmospheres have become available in the recent years. They are based on the idealized assumptions of spherical symmetry, stationarity and radiative equilibrium. The satisfactory agreement between calculated and observed Wolf-Rayet spectra suggests that this "standard model" is basically adequate for describing real WR atmospheres and hence can be applied for their quantitative spectral analyses. By the application of these models, the fundamental parameters have been determined meanwhile for the majority of the known Galactic WR stars. Most WN stars populate a vertical strip in the Hertzspung-Russell diagram at effective temperatures of $\approx 35 \mathrm{kK}$, the luminosities ranging from $10^{4.5}$ to $10^{5.9} L_{\odot}$. Only three WN stars of earliest subtype, other early-type WN stars if they have strong lines, and the WC stars are hotter. The chemical compositions of the WR atmospheres correspond to nuclear-processed material (WN: hydrogen burning in the CNO cycle; WC: helium burning). Hydrogen is depleted but still detectable in the cooler members of the WN subclass. Quantitatively, the hydrogen abundances show an interesting correlation with the luminosity which can be compared with the predictions from evolutionary calculations.
\end{abstract}

Key words: stars: Wolf-Rayet - expanding atmospheres - mass-loss - fundamental parameters - element abundances

\section{Model atmospheres}

Wolf-Rayet spectra are characterized by bright and broad emission lines, in some cases accompanied by blue-shifted absorption features (P-Cygni profiles). Their spectral appearance is the consequence of a strong stellar wind, which provides a large line-emitting volume and broadens the lines by Doppler shift due to radial expansion. This explanation for WR spectra was suggested long ago by Beals (1929) and is nowadays widely accepted, while Underhill (e.g., 1991) still favors an alternative model.

Spectral analyses of WR stars, i.e., the determination of their parameters and atmospheric composition, requires adequate models which account for the extreme non-LTE situation, the spherical symmetry and the radial expansion. A first approach to the quantitative modelling of WR spectra was made by Castor \& Van Blerkom (1970) who treated the non-LTE radiation transfer for one "representative" point in the WR atmosphere by means of photon escape probabilities ("Sobolev approximation"). While they applied their method to the HeII lines of two WN6 stars, it was used for various abundance determinations for $\mathrm{H}$ and $\mathrm{CNO}$ elements by, e.g., Willis \& Wilson (1978), Smith \& Willis (1982, 1983), Nussbaumer et al. (1979), Nugis (e.g., 1982a,b; 1991). Another class of one-point models applied nebular ap- 
proximations (recombination theory) to WR atmospheres (Smith \& Hummer 1988, Torres 1988). The results from these one-point model analyses remained in parts contradictory.

Today we know that in most WR atmospheres the ionization varies strongly with the radius in the sense that recombination proceeds towards outer layers. Thus different spectral lines are formed in different parts of the atmosphere, making one-point models obsolete.

Stratified non-LTE models for expanding atmospheres were developed during the last decade independently by Hillier and by Hamann et al. ("Kiel group"). Both codes can treat the line formation transfer in the comoving frame (i.e., not relying on Sobolev's approximation). Hillier ( $c f .1990)$ solves the full set of equations by linearization, suppressing those terms coupling more than (optionally) one, three or five spatial points. The Kiel group has adapted the "iteration with approximate lambda operators" (Hamann 1985, 1986, 1987; Hamann \& Wessolowski 1990; Koesterke et al. 1992) to the situation of spherical expansion. In the "local" version, both iteration methods are presumably equivalent. Many further details are handled differently in both codes. Nevertheless both codes agree remarkably well in their results.

The available model atmospheres for WR stars (both versions) are based on simplifying assumptions called the "standard model", which represents the most-idealized form of an expanding atmosphere. Spherical symmetry, together with stationarity and homogeneity, implies that the equation of continuity relates the velocity field $v(r)$ and the density structure $\rho(r)$ for a given mass-loss rate $\dot{M}$. No self-consistent hydrodynamic calculations for WR atmospheres are possible yet, as the acceleration mechanism is not even identified. Instead, the velocity field must be pre-specified ad hoc, and one usually assumes the law $v(r)=v_{\infty}\left(1-\frac{r_{0}}{r}\right)^{\beta}$, where the terminal velocity $v_{\infty}$ is a free parameter, and $\beta=1$ seems to be the best choice for the exponent in most cases. Fortunately, the results are often (i.e., for dense winds) not sensitive to details of the velocity law, as the lines are formed at large radii where the terminal velocity is almost approached. The electron temperature stratification follows from the assumption of radiative equilibrium.

Both codes (from Hillier and from Hamann's group, respectively) have achieved about the same degree of sophistication in their latest versions. Complex model atoms for, $\epsilon . g ., \mathrm{N}$ or $\mathrm{C}$ can be treated with typically $\approx 100$ energy levels and $\approx 1000$ line transitions. Low-temperature dielectronic recombination (LTDR) is accounted for in an approximate way. Frequency redistribution of line photons by electron scattering is included in the Formal Integral. The neglect of blanketing effects from "metal" lines is one deficiency of the present models and may lead to an over-estimate of the WN luminosities by a factor of two (Schmutz 1994).

With the "standard model" the observed spectra can be reproduced satisfactorily. However, certain minor discrepancies are notoriously encountered. 
The absorption feature of P-Cygni profiles are often predicted deeper than observed. The extended wings of strong emission lines which are caused by electron-scattering frequency redistribution are often weaker observed than calculated. The fit of lines from very high and rather low ions (e.g., O VI and $\mathrm{C}$ III in a WC5 star) requires models with slightly different parameters. All these problems might reflect inhomogeneities ("clumping") in the atmosphere, as also indicated by the observed profile variabilities (e.g., Moffat et al. 1988). A study by Hillier (1984), and simple extrapolation from the homogeneous "standard models" suggest that the neglect of clumping may lead to an over-estimate of mass-loss rates by perhaps a factor of two. The effective temperatures and hence the luminosities are probably not affected significantly. The mass-loss rates derived from radio-emission measurements agree well with those from spectral line analyses (Schmutz et al. 1989), which is another argument against clumping being very effective. However, these questions deserve further investigation.

X-ray emission has been observed from a couple of WR stars with EINSTEIN and ROSAT. Apart from possible binarity, this cannot be explained within the "standard model". Baum et al. (1992) constructed a semi-empirical two-component model accounting additionally for hot, shocked gas. The observed count rates can be explained with that model, and even the spectral distribution of the X-rays as observed with ROSAT PSPC can be reproduced ( $c f$. Wessolowski et al., these proceedings). These studies allow the important conclusion that the "standard model", although neglecting the $\mathrm{X}$-ray emitting hot component, remains valid for describing the visual and UV spectrum. The number of ionizing $X$-ray photons is too small to compete with the high recombination rates present in the relatively dense WR winds.

\section{Analyses and results}

Hillier's models have been applied so far mainly for pilot studies: He and $\mathrm{H}$ lines of the WN5 prototype WR 6 (Hillier $1987 \mathrm{a}, \mathrm{b}$ ), $\mathrm{N}$ and $\mathrm{C}$ lines in the same star (Hillier 1988), and carbon lines in the WC6 prototype WR 111 (Hillier 1989). Systematic spectral analyses of WN stars (accounting partly for He, $\mathrm{H}, \mathrm{C}, \mathrm{N}, \mathrm{O}, \mathrm{Si}$ ) are presently performed with Hillier's code at the University College London (Crowther et al. 1994a,b and in preparation).

The Kiel group started with pure-helium models, which were tested in a couple of pilot analyses (e.g., Hamann et al. 1988). A whole grid of models was published (Wessolowski et al. 1988) and applied for coarse analyses of 30 Galactic WR stars (Schmutz et al. 1989). This study was extended to the vast majority ( 53 of 71 ) of the known Galactic single WN stars (Hamann et al. 1993a). Recently the Galactic sample has been nearly completed, including many detailed analyses and quantitative determinations of the hydro- 


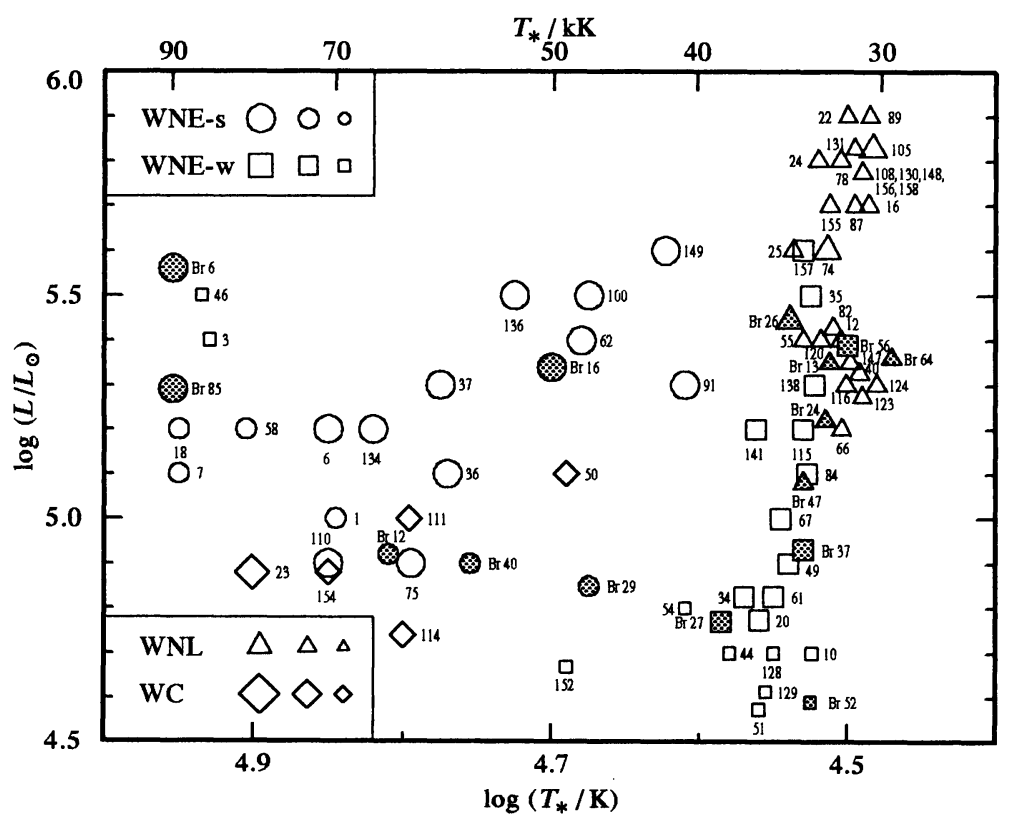

Fig. 1. Empirical HRD of the WR stars. The Galactic stars are identified by their WR numbers (van der Hucht et al. 1981), while Br labels and shaded symbols refer to LMC objects (Breysacher 1981). The spectral subgroups are distinguished by different symbols (see insets), while their size indicates whether the mass-loss rate is large $\left(>10^{-4.2} M_{\odot} / \mathrm{yr}\right)$, small ( $\left.<10^{-4.8} M_{\odot} / y r\right)$, or intermediate. Data taken from Hamann et al. (1993a and in preparation) for Galactic WN, Koesterke etal. (1991) for LMC WN, and Koesterke et al. (submitted) for WC stars.

gen abundance (Hamann et al., in preparation). $19 \mathrm{WN}$ stars in the Large Magellanic Cloud (LMC) were analyzed by Koesterke et al. (1991). For a pilot study with nitrogen we selected the WN6 star WR 136 (Hamann et al. 1993b). The carbon models were tested in Kiel with the same star WR 111 (Hamann et al. 1992) Hillier had used. A whole grid of WC models was published by Koesterke et al. (1992). A comprehensive analysis of 25 Galactic WC5-8 stars with individual $\mathrm{He}+\mathrm{C}$ model calculations has been completed recently (Koesterke et al. , submitted).

The analyses of WR stars performed by the Kiel group lead to the empirical Hertzsprung-Russell diagram (HRD) shown in Fig. 1. The WNL stars (late WN subtypes: WN7 ...WN9) are relatively cool $\left(T_{*} \approx 35 \mathrm{kK}\right)^{*}$ and populate a strip between $\log L / L_{\odot}=5.2 \ldots 5.9$. Stars with WNE-w spectra (early WN subtypes - WN2 ... WN6 - and weak lines) form an extension of that strip $\left(T_{*} \approx 35 \ldots 40 \mathrm{kK}\right)$ to lower luminosities down to $\log L / L_{\odot}=4.5$. The WNE-s stars (early subtypes, strong lines) are distinctively hotter $\left(T_{*}>50 \mathrm{kK}\right)$, have

* $T_{*}$ is the effective temperature referring to the radius of the hydrostatic core radius $R_{*}$. For an exhaustive discussion of the effective temperature definitions see Hamann (1994) 


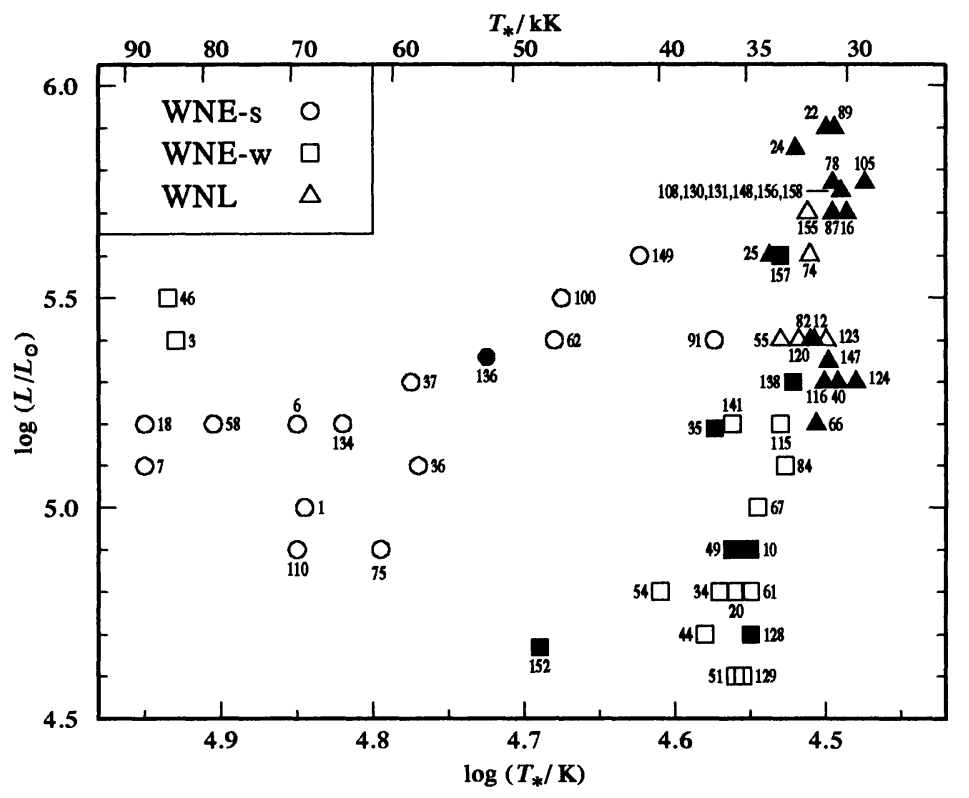

Fig. 2. HRD of the Galactic WN stars indicating the presence (filled symbols) or absence (open symbols) of hydrogen. The stars are again identified by their WR numbers, while the symbol shape refers to the spectral subclass (see inset). From Hamann et al. (in preparation)

the largest mass-loss rates, and scatter between $\log L / L_{\odot}=4.9$ and 5.6. The three Galactic WN stars of earliest subtype (WR2, WR 3 and WR 46) which do not show any He I lines constitute a separate class. Analyses of their nitrogen spectra reveal that they are very hot. Preliminary results for WR 3 and WR 46 from Wessolowski $\epsilon t$ al. (in prep.) are included in Figs.1-3.

The WN stars of the LMC ("Br" labels) share their locations with their Galactic counterparts. This constrains the metallicity dependence of the evolutionary formation of WR stars ( $c f$. Sect.3).

First results from recent analyses of Galactic single WC stars by means of He-C-models are also included in Fig. 1. Represented are five stars of intermediate subtype (WC.5-8) with known distances. These WC stars obviously populate the same region in the HRD as the WNE-s stars.

Hydrogen in the helium-dominated WN atmospheres is not easy to recognize, because all $\mathrm{HI}$ lines coincide with He II lines. A qualitative assessment of the presence or absence of hydrogen has been based on the comparison between the unblended and blended (with $\mathrm{H}$ I Balmer lines) members of the He II Pickering series (Conti et al. 1983, Hamann et al. 1993a). The results from that method have been confirmed meanwhile for many stars by detailed analyses with profile fits (e.g., Hamann et al. in preparation). The occurrence of hydrogen is found to correlate with the HRD position (Fig. 2), 


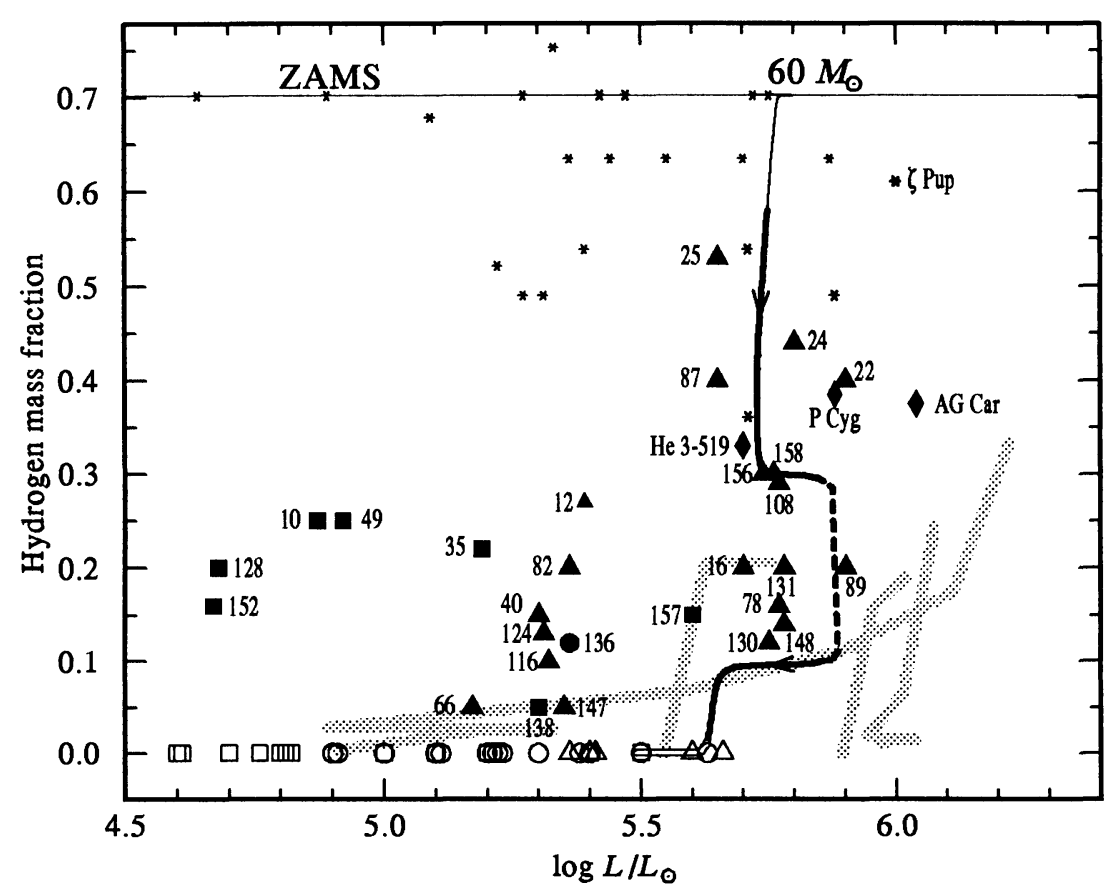

Fig. 3. Hydrogen abundance vs. luminosity for Galactic WN stars (labels: WR numbers). Open symbols crowding at zero hydrogen abundance indicate the luminosities of those WN stars showing no hydrogen. The symbol shapes encode the WN subclasses ( $c f$. inset of Fig. 2). Three LBVs (black diamonds) and several OB stars ( $\star$ ) are also represented. For the discussion of the evolutionary status (cf. Sect. 3), shaded lines represent the "WNL" phase of "standard" evolutionary tracks (most recent version by Meynet et al. 1994) for ZAMS masses of $120,85,60$ and $40 M_{\odot}$ (track for largest mass starts with largest luminosity) and solar metallicity. An alternative track for evolution with pulsation-driven mass-loss (from Langer et al. 1994) is represented from the ZAMS $\left(M=60 M_{\odot}\right)$ through the $\mathrm{O}$ and Of phase (thin line), the "WNL" phase (thick line) interrupted by an LBV excursion (dashed line) until the "WNE" phase (double line). See Langer et al. 1994 for more references and details.

as it is restricted to the lowest effective temperatures. But the discrimination does not strictly follow the spectroscopic classification as WNL or WNE. One part of the WNE-w stars shows hydrogen like most WNL stars, while the other part is hydrogen-free like the WNE-s group. Furthermore, there are a few exceptions from the rule: the WN6 "prototype" (!) WR 136 shows hydrogen, while five WNL are hydrogen-free.

For almost all Galactic WN stars the hydrogen abundances have been now determined quantitatively (Hamann et al. in prep., including results from Crowther et al. $1994 \mathrm{~b}$ and in preparation). Plotting the hydrogen mass fraction vs. luminosity reveals an interesting pattern (Fig. 3). Below a luminosity of $10^{5.6} L_{\odot}$ one finds only hydrogen abundances in the range $0 \ldots 0.25$. Above $10^{5.7} L_{\odot}$ there exist no hydrogen-free $W N$ atmospheres at all. Instead, the 


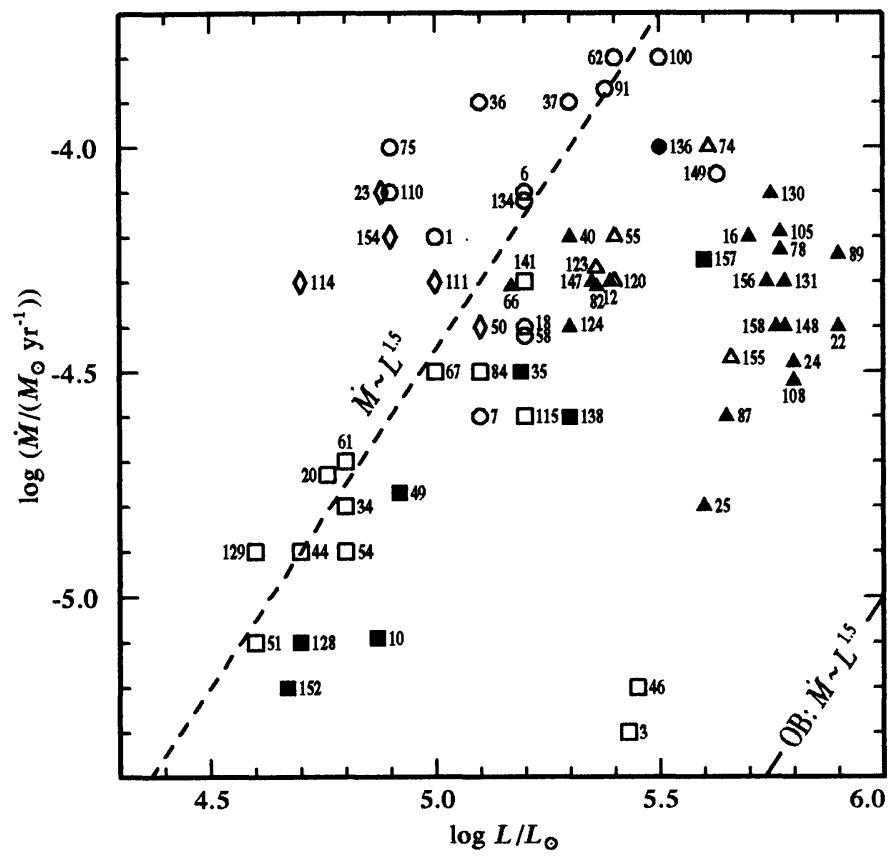

Fig. 4. Mass-loss rates of Galactic WR stars vs. their luminosities. Different symbol shapes distinguish the spectral subclasses ( $c f$. inset of Fig. 2), while filled or open symbols indicate whether hydrogen is detectable or absent, respectively. The dashed line indicates the relation $\log \dot{M} /\left(M_{\odot} \mathrm{yr}^{-1}\right)=1.5 \log L / L_{\odot}-11.95$ as a tentative fit to the hydrogen-free stars.

observed hydrogen mass fractions for the most luminous WN stars range from 0.15 to 0.53 .

The chemical compositions of WN atmospheres, as far as analyzed yet, are obviously the result of (partial or complete) hydrogen burning in the CNO cycle. Helium is dominating, while hydrogen is depleted. Nitrogen contributes $1-2 \%$ to the mass, while carbon is underabundant (Hillier 1988; Hamann et al. 1993b; Crowther et al. 1994a,b and in preparation). WC stars obviously present material which was partly processed by the $3 \alpha$-reaction. The intermediate subtypes (WC5-8) analyzed so far by means of stratified models (Hillier 1989; Hamann et al. 1992; Koesterke et al. submitted) show carbon-to-helium ratios between 0.25 and 1.5 (by mass), which is in the range predicted by the standard evolutionary models. While Koesterke et al. (submitted) cannot find any correlations between the $\mathrm{C} / \mathrm{He}$ ratio and other parameters, Smith \& Hummer (1988) obtained a connection between abundances and subtype from their analyses with recombination theory. The determination of oxygen abundances in WC and WO stars should be subject to future work.

The mass-loss rates of the Galactic WR stars are plotted vs. luminosities 
in Fig. 4. Although there is a large scatter, the data for the hydrogen-free stars (open symbols) may be roughly represented by $\dot{M} \propto L^{1.5}$. Interestingly, this line has the same slope (1.5) as found for OB stars, while it lies at higher $\dot{M}$ by about 2.0 dex. Most WC stars lie to the left of this relation. The WNE-w stars of earliest subtype (WR 3, WR 46) again form their own class. The WN stars with hydrogen (filled symbols, mainly WNL) have generally smaller $\dot{M}$ than the relation indicated.

\section{Conclusions}

The evolution of massive stars under so-called "standard assumptions" has been calculated by the Geneva group in a series of papers. Previous versions of these tracks (e.g., Schaller et al. 1992) predicted a lower luminosity limit for WN stars which was in conflict with the empirical results. In the most recent version, Meynet et al. (1994) now assumed stronger mass-loss during the pre-WR phases. These tracks still predict a minimum ZAMS mass for WR progenitors ( 25 to $40 M_{\odot}$ ), but very massive stars $\left(e . g ., M_{\mathrm{ZAMS}}=120 M_{\odot}\right.$ ) now experience a dramatic decline of luminosity during their WN stage. Thus, the least luminous WN stars could originate from the most massive progenitor. Only a population synthesis might reveal whether this could explain the observed luminosity distribution. For the LMC the new "standard" tracks (for which a scaling of $\dot{M}$ with metallicity as $Z^{1 / 2}$ is adopted) still predict a lower luminosity limit for WN stars which is strongly in conflict with the empirical data ( $c f$. Fig. 1). The surface hydrogen abundances predicted by the "standard" evolutionary calculations are not in good agreement with the values observed in the Galactic sample (Fig. 3).

An alternative evolutionary scenario has been proposed recently by Langer et al. (1994), accounting for pulsation-driven mass-loss caused by the theoretically discovered "dynamical strange-mode instabilities" (Kiriakidis et al. 1993, Glatzel et al. 1993). A pilot calculation starting with $M_{\text {ZAMS }}=$ $60 M_{\odot}$ ) yields an evolutionary sequence $\mathrm{O} \rightarrow \mathrm{Of} \rightarrow \mathrm{H}$-rich $\mathrm{WN} \rightarrow \mathrm{LBV} \rightarrow \mathrm{H}-$ poor $\mathrm{WN} \rightarrow \mathrm{H}$-free $\mathrm{WN} \rightarrow \mathrm{WC} \rightarrow \mathrm{SN}$. A favorable feature of this scenario is the prediction of a first "WNL" stage with rather high hydrogen abundance, followed by the LBV phase and a subsequent second "WNL" stage with less hydrogen, qualitatively in accordance to the empirical data (cf. Fig. 3).

Close-binary evolution could produce WR stars from less massive progenitors than single-star evolution. Double-lined binaries have been generally excluded from the spectral analyses because of the problem of spectrum decomposition. Another $15 \mathrm{WN}$ stars and, remarkably, only one (even uncertain) WC star are suspected as single-lined spectroscopic binaries (SB1). Pointing out these SB1 stars in the empirical HRD (Fig.1) reveals no systematic trends. This does not corroborate the hypothesis that WN stars of low luminosity were preferably formed in close-binary systems. 


\section{References}

Baum, E., Hamann, W.-R., Koesterke, L., Wessolowski, U. 1992, A\&A 266, 402

Beals, C.S. 1929, MNRAS 90, 202

Breysacher, J. 1981, A $\mathscr{E} A$ Suppl. 43, 203

Castor, J.I., Van Blerkom, D. 1970, $A p J 161,485$

Conti, P.S., Leep, E.M., Perry, D.N. 1983, ApJ 268, 228

Crowther, P., Hillier, D.J., Smith, L.J. 1994a, Sp. Sci. Rev. 66, 271

Crowther, P., Hillier, D.J., Smith, L.J. 1994b, $A \& A$ in press

Glatzel, W., Kiriakidis, M., Fricke, K.J. 1993, MNRAS 262, L7

Hamann, W.-R. 1985, $A \& A$ 148, 364

Hamann, W.-R. 1986, $A \& B A 160,347$

Hamann, W.-R. 1987, in: W. Kalkofen (ed.), Numerical Radiative Transfer (Cambridge: CUP), p. 35

Hamann, W.-R. 1994, Sp. Sci. Rev. 66, 237

Hamann, W.-R., Schwarz, E. 1992, $A \cup A$ 261, 523

Hamann, W.-R., Wessolowski, U. 1990, $A \varangle A$ 227, 171

Hamann, W.-R., Schmutz, W., Wessolowski, U. 1988, $A \& A$ 194, 190

Hamann, W.-R., Leuenhagen, U., Koesterke, L., Wessolowski, U. 1992, $A \mathscr{E} A$ 255, 200

Hamann, W.-R., Koesterke, L., Wessolowski, U. 1993a, $A \& A$ 274, 397

Hamann, W.-R., Wessolowski, U., Koesterke, L. 1993b, $A \varepsilon^{\varepsilon} A$ 281, 184

Hillier, D.J. 1984, $A p J$ 280, 744

Hillier, D.J. 1987a, ApJ Suppl. 63, 947

Hillier, D.J. 1987b, ApJ Suppl. 63, 965

Hillier, D.J. 1988, $A p J$ 327, 822

Hillier, D.J. 1989, $A p J$ 347, 392

Hillier, D.J. 1990, $A \& A$ 231, 116

van der Hucht, K.A., Conti, P.S., Lundström, I., Stenholm ,B. 1981, Sp. Sci. Rev. 28, 227

Kiriakidis, M., Fricke, K.J., Glatzel, W. 1993, MNRAS 264, 50

Koesterke, L., Hamann, W.-R., Schmutz, W., Wessolowski, U. 1991, A\&A 248, 166

Koesterke, L., Hamann, W.-R., Wessolowski, U. 1992, $A \& A$ 261, 535

Langer, N., Hamann, W.-R., Lennon, M., Najarro, F., Pauldrach, A.W.A., Puls, J. 1994, $A \mathscr{E} A$ in press

Meynet, G., Maeder, A., Schaller, G., Schaerer, D., Charbonnel, C. 1994, A 6 A Suppl. 103,97

Moffat, A.F.J., Drissen, L., Lamontagne, R., Robert, C. 1988, ApJ 334, 1038

Nugis, T.: 1982a,b, in: C.W.H. de Loore \& A.J. Willis (eds.), Wolf-Rayet stars: Observations, Physics, Evolution, Proc. IAU Symp. No. 99 (Dordrecht: Reidel), p. 127, p. 131

Nugis, T. 1991, in: K.A. van der Hucht \& B. Hidayat (eds.), Wolf-Rayet Stars and Interrelations with Other Massive Stars in Galaxies, Proc. IAU Symp. No. 14.3 (Dordirecht: Kluwer), p. 75

Nussbaumer, H., Schmutz, W., Smith, L.J., Willis, A.J., Wilson, R. 1979, in: A.J. Willis (ed.), The First Year of IUE (London: UCL), p. 259

Schaller, G., Schaerer, D., Meynet, G., Maeder, A. 1992, A EA Suppl. 06, 269

Schmutz, W. 1994, Sp. Sci. Rev. 66, 253

Schmutz, W., Hamann, W.-R., Wessolowski, U. 1989, A\&A 210, 236

Smith, L.F., Hummer, D.G. 1988, MNRAS 230, 511

Smith, L.J., Willis, A.J. 1982, MNRAS 201, 451

Smith, L.J., Willis, A.J. 1983, A E A Suppl. 54, 226

Torres, A.V. 1988, ApJ 325, 759

Underhill, A.B. 1991, ApJ 383, 729

Wessolowski, U., Schmutz, W., Hamann, W.-R. 1988, $A$ EiA 194, 160

Willis, A.J., Wilson, R. 1978, MNRAS 182, 559 


\section{DISCUSSION:}

van Kerkwijk: The two WNE-w without HeI that were analysed on the basis of the $\mathrm{N}$ lines, are in a very different position in the HRD than the WNE-w stars with HeI that were analysed on the basis of the He lines. Does the position of the latter change when analysed on the basis of the $\mathrm{N}$ lines?

Hamann: Hopefully not. It seems that the different parameters obtained for those earliest-type WN stars (WR 2, 3 and 46) are not due to different methods of analysis. Indeed, inspection of the nitrogen spectrum reveals a qualitative jump when comparing with the most similar spectrum (WR 152) of the "normal" WNE-w sequence. However, this point is still preliminary. Crowther: My comment is that it is not obvious how we can reconcile the observed (spatial, spectral etc.) differences between many 'H-rich' WNL stars and LBVs - so no observational evidence exists that there is an evolutionary link between these stars.

Hamann: The evolutionary sequence $\mathrm{O} \rightarrow \mathrm{H}$-rich WNL $\rightarrow \mathrm{LBV} \rightarrow \mathrm{H}$-poor WNL $\rightarrow$ WNE $\rightarrow$ WC predicted theoretically by Langer et al. (1994) has been supported yet only from the observed HRD positions and hydrogen abundances.

Cherepashchuk: Did you try to find some correlation between values of mass loss rate and effective temperatures?

Hamann: We looked for any correlations between the different empirical parameters of the galactic WN stars, but $T_{*}$ and $M$ are not well correlated. There is a correlation between the mass flux (i.e. $\mathrm{M} \mathrm{R}^{2}$ ) and $\mathrm{T}_{*}$ for the hydrogen-free $\mathrm{WN}$ stars as pointed out by $\mathrm{W}$. Schmutz in an earlier paper, which is equivalent to the $M \propto L^{1.5}$ relation that $I$ have shown in my corresponding figure.

Maeder: I fully agree with you that the Xs (hydrogen content by mass) vs $\log \mathrm{L} / \mathrm{L} \odot$ is a major constraint for WN stars. Indeed, the comparisons are much better when made for models with a metallicity higher than solar. However, even so it is difficult to escape to the conclusion that mixing is necessary in the interior of massive stars.

Lindsey Smith: I am very happy to see a correlation between high mass loss and low $\mathrm{H} / \mathrm{He}$. Since the $\epsilon$-mechanism instability strongly appeals to me as relevant in WR mass loss ratios: the presence of $\mathrm{H}$ on the surface dramatically reduces the instability because of the associated extension of the atmosphere.

Moffat: This morning we heard about clumping from I. Antokhin. How will this impact in the determination of your WR parameters? Note that direct observation of mass loss in V444 Cygni shows that due to clumping (probably), the smooth-wind based mass loss rate (IR, radio) must be reduced by at least a factor 3 (St Louis et al. 1993).

Hamann: I am prepared for that question. Indeed, there are indications for clumping also from our spectral fits: electron-scattering line wings are sometimes weaker observed than predicted, P-Cygni type absorption features (e.g. of He I 5876) are weaker observed than predicted, and the span of observed ionization stages (e.g. OVI to CIII in WR111) are not fully reproducible with the same model. All these discrepancies suggest clumping with a density contrast of, very roughly, a factor of three.

In a primitive model, as a first step to a more elaborate modelling, we might assume that all line emission comes from "clumps" which have a volume filling factor $f$, while the emission equivalent widths are taken from that standard model with the clump density (i.e. $1 / \mathrm{f} \mathrm{times}$ enhanced), but scaled down as W $\lambda \times \mathrm{ff}$. When analysing a typical WN star (e.g. WR 78, WN7), the following results would be obtained in dependence of the assumed $\mathrm{f}$ : 


$\begin{array}{ccc}\mathrm{f}^{1} & \mathrm{~T} / \mathrm{NK} & \dot{\mathrm{M}}^{\circ} \dot{\mathrm{M}}_{\text {tdd }} \\ 1 & 32 & 1 \\ 2 & 33 & 0.7 \\ 3 & 33.5 & 0.6 \\ 4 & 34 & 0.6 \\ 5 & 36 & 0.7 \\ 6 & 42 & 1.1 \\ 7 & 50 & 2.0 \\ 8 & 56 & 2.5\end{array}$

Thus, moderate clumping with a plausible filling factor of, say, $1 / 3$ would reduce the derived mass-loss rates by a factor of 2 , while the temperature and all other parameters are not significantly affected. Of course, we must investigate this further with more elaborate "clumping models".

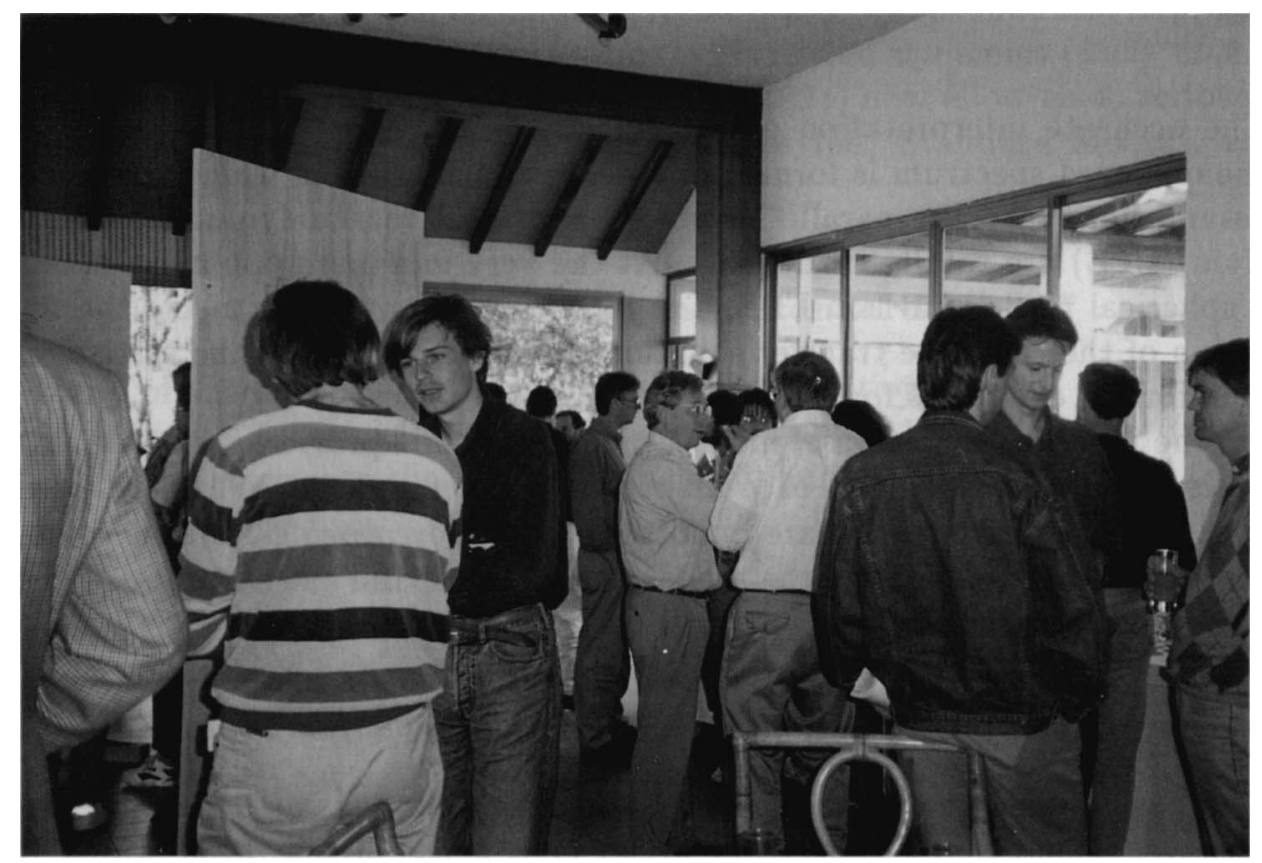

van Kerkwijk, De Greve, van der Hucht, Berghöfer, Hamann 